\title{
Primary infection with human herpes virus type 6, post-pediatric liver transplantation - a pathogen to remember
}

Adi Pappo-Toledano ${ }^{1}$, Sara Dovrat ${ }^{2}$, Zvia Soufiev², Yael Mozer-Glassberg' ${ }^{3,4}$, Irit Krause ${ }^{1,4}$, Eran Rom ${ }^{1,4}$, Raanan Shamir ${ }^{3,4}$, Efraim Bilavsky ${ }^{1,4}$

${ }^{1}$ Department of Pediatrics C, Schneider Children's Medical Center, Petah Tiqva, Israel ${ }^{2}$ Central Virology Laboratory, Public Health Services Israel Ministry of Health, Chaim Sheba Medical Center, Tel Hashomer, Israel ${ }^{3}$ Institute for Gastroenterology, Nutrition and Liver Diseases, Schneider Children's Medical Center, Petah Tiqva, Israel ${ }^{4}$ Sackler Faculty of Medicine, Tel Aviv University, Tel Aviv, Israel

\section{BACKGROUND}

In recent years, liver transplantation (LT) has become a wellaccepted therapeutic modality for children with end stage liver disease, with transplantation surgery being performed at a younger age. Infection with human herpes virus 6 (HHV-6) occurs in most children within the first two years of life, therefore, data on primary HHV-6 infection in pediatric liver transplant recipients is scarce.

\section{OBJECTIVE}

To describe the course of primary HHV-6 infection after pediatric LT.

\section{METHODS}

Medical files, between the years 2015-2016, of post-LT pediatric patients with suspected primary HHV-6 infection were reviewed. Clinical and laboratory data for enrolled cases were evaluated. Primary infection was defined as DNAemia in children who were seronegative prior to transplantation or seroconversion from negative to positive IgG post- transplantation.

\section{RESULTS}

Four cases of primary HHV-6 (type B) infection were identified amongst the 26 children who had undergone LT at our center during the study period. All patients were $<1$ year old and presented with fever, hepatitis and elevated inflammatory markers, most $(75 \%)$ within a short period post-transplantation. All were initially treated with empiric antibiotics for a suspected bacterial infection and 3 underwent liver biopsy, one showing signs of rejection. Three were treated with antiviral therapy with a gradual resolution of symptoms.

\section{Characteristics of children with primary HHV-6 infection post-liver transplantation}

\begin{tabular}{|c|c|c|c|c|c|c|c|c|c|c|c|}
\hline No. & $\begin{array}{l}\text { Age } \\
\text { mo/ } \\
\text { sex }\end{array}$ & $\begin{array}{l}\text { Indication } \\
\text { for } \\
\text { transplant }\end{array}$ & $\begin{array}{l}\text { Living or } \\
\text { Deceased } \\
\text { Donor }\end{array}$ & $\begin{array}{l}\text { Time to } \\
\text { symptoms }\end{array}$ & $\begin{array}{l}\text { HHV-6 } \\
\text { detection }\end{array}$ & Seroconversion & $\begin{array}{l}\text { Clinical and } \\
\text { laboratory } \\
\text { presentation }\end{array}$ & Type & $\begin{array}{l}\text { Rejection } \\
\text { or PTLD }\end{array}$ & Treatment & $\begin{array}{l}\text { CMV } \\
\text { Prophylaxis }\end{array}$ \\
\hline 1 & $6 / \mathrm{F}$ & BA & Living & POD23 & $\begin{array}{l}\text { Plasma and } \\
\text { whole } \\
\text { blood PCR }\end{array}$ & Yes & $\begin{array}{l}\text { Fever, } \\
\text { Elevated LF }\end{array}$ & B & $\begin{array}{l}\text { Acute } \\
\text { cellular } \\
\text { rejection }\end{array}$ & $\begin{array}{l}\text { Abx, GCV, IV } \\
\text { steroids }\end{array}$ & VGCV \\
\hline 2 & $8 / \mathrm{M}$ & MSUD & Deceased & POD15 & $\begin{array}{l}\text { Plasma and } \\
\text { whole } \\
\text { blood PCR }\end{array}$ & Yes & $\begin{array}{l}\text { Fever, } \\
\text { Diarrhea, } \\
\text { Elevated LF }\end{array}$ & B & no & Abx, GCV & VGCV \\
\hline 3 & $8.5 / \mathrm{M}$ & BA & Living & POD242 & $\begin{array}{l}\text { Plasma and } \\
\text { whole } \\
\text { blood PCR }\end{array}$ & Yes & $\begin{array}{l}\text { Fever, } \\
\text { Pharyngitis, } \\
\text { Elevated LF }\end{array}$ & B & no & $A b x$ & Non \\
\hline 4 & $6 / \mathrm{M}$ & BA & Living & POD54 & $\begin{array}{l}\text { Plasma and } \\
\text { whole } \\
\text { blood PCR }\end{array}$ & Yes & $\begin{array}{l}\text { Fever, } \\
\text { Pharyngitis, } \\
\text { Rash, } \\
\text { Diarrhea, } \\
\text { elevated LF }\end{array}$ & B & $\begin{array}{l}\text { PTLD, no } \\
\text { rejection }\end{array}$ & $\begin{array}{l}\text { Abx, VGCV } \\
\text { twice daily }\end{array}$ & VGCV \\
\hline
\end{tabular}

No. number; PTLD, post-transplant lymphoproliferative disease; BA, biliary atresia; POD, post-operative day; LF, liver functions; ABX, antibiotics; GCV, Ganciclovir; VGCV, Valganciclovir; MSUD, maple syrup urine disease.

\section{DISCUSSION}

Primary HHV-6 should be taken into account in young children shortly after LT, especially when presenting with fever and elevated liver enzymes. Treatment with anti-viral therapy should be considered.

\section{CONCLUSION}

In young infants post-LT, a high index of suspicion may promote early detection of HHV6 primary infection and prevent serious complications. 